\title{
Real-Time-Photogrammetrie in der Urologie
}

\author{
II. Erste klinische Erfahrungen mit einem experimentellen \\ Sichtprüfsystem („PRIAPUS“) in der Diagnostik erektiler Dysfunktion
}

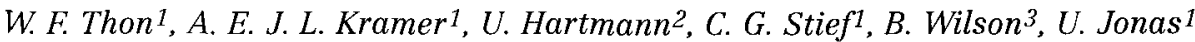 \\ ${ }^{1}$ Urologische Klinik, Med. Hochschule Hannover \\ 2 Zentrum Psychologische Medizin, Med. Hochschule Hannover \\ 3 VEXCEL Corporation, Boulder, Colorado, USA
}

\section{Zusammenfassung}

Die Beurteilung der Erektionsfähigkeit während audiovisueller sexueller Stimulation und/oder intrakavernöser Schwellkörperinjektionstestung ist abhängig von der subjektiven Einschätzung des Untersuchers. Die kontinuierliche Aufzeichnung der Penisrigidität mit Hilfe dem Penis angelegter Meßschleifen ermöglicht eine objektive Dokumentation der Rigidität während der Untersuchung, erlaubt aber keine Aussage über eine Längen-, Durchmesser- oder Volumenzunahme des Penis oder Deviation des Gliedes bei Erektion. In Experimenten mit Penismodellen konnten wir demonstrieren, daß ein von uns konzipiertes Sichtprüfsystem („PRIAPUS“) exakt und reproduzierbar berührungsfrei Länge, Durchmesser und Volumen mißt (7). Die erste praktische Anwendung dieses Real-Time-Photogrammetrie-Systems im Vergleich mit der RigiScan ${ }^{\circledR}$ Aufzeichnung bei 30 Probanden zeigte eine signifikante Korrelation der Rigidität mit der Zunahme des maximalen Penisdurchmessers und des Volumens. Bei 11 Probanden mit einer Rigidität $>70 \%$ wurde die physiologische Kurvatur des erigierten Penis mit 0,05 Grad $/ \mathrm{cm}$ bestimmt.

\author{
Real-Time Photogrammetry in Urology \\ II. Early Clinical Experience with an \\ Experimental Image Examination \\ System in the Diagnosis of Erectile \\ Dysfunction
}

Assessment of erectile capabilities by inspection or palpation during audiovisual sexual stimulation and/or after intracavernous injection of vasoactive drugs depends upon the examiner's subjective estimation. Penile rigidity recordings with the help of measuring loops attached to the penis objectively document rigidity during examination, but do not provide information about changes in penile length, diameter volume and curvature. In an experimental set-up with penile models we have already demonstrated that the penile measurements obtained by an image examination system ("PRIAPUS") using photogrammetric tools are exact and reproducible (7). The first practical use of the system with 30 volunteers showed a significant correlation of rigidity and increase of maximum diameter and volume as compared to RigiScan ${ }^{\circledR}$ recordings. In 11 volunteers with a rigidity $>70 \%$ we recorded a physiological curvature of the erect penis of 0.05 degrees $/ \mathrm{cm}$.

\section{Key words}

Photogrammetry - Erectile dysfunction Image examination system
Bei der Diagnostik der erektilen Dysfunktion mit Schwellkörperinjektionstestung und Inspektion und Palpation des Penis oder Rigiditätsaufzeichnung mit Hilfe dem Penis angelegter Meßschleifen dringt der Untersucher in einen sehr intimen Bereich des Patienten vor. Allein aufgrund der Untersuchungssituation ist mit einer negativen Beeinflussung der aufgezeichneten Erektionsfähigkeit zu rechnen. Die Beurteilung der Penisformveränderung des flakziden und erigierten Penis obliegt der subjektiven Einschätzung des Untersuchers. Das von

Akt. Urol. 24 (1993) 95-99

(C) Georg Thieme Verlag Stuttgart · New York
Bradley et al. 1985 (2) eingeführte RigiScan ${ }^{\circledR}$-Gerät ermöglicht durch zwei dem Penis angelegte Meßschleifen neben der Tumeszenzaufzeichnung (Umfangsänderung) auch eine Registrierung der zirkumferenten Penisrigidität, die linear mit der axialen Rigidität und dem intrakavernösen Druck korreliert (4). Trotz akkurater Aufzeichnung nokturner peniler Tumeszenz und Rigidität (NPTR) über mehrere Nächte, kann eine Diskrepanz zwischen diesem Ergebnis und der Erektionsfähigkeit bei entsprechender sexueller Stimulation bestehen $(1,3)$. Aus diesem Grunde führen wir die von Giesbers et al. (5) beschriebene Real-Time-RigiScan ${ }^{\circledR}$-Aufzeichnung während audiovisueller sexueller Stimulation (AVSS) und intrakavernöser 
Injektionstestung durch (6). Die von zahlreichen Patienten glaubhaft berichtete stärkere Erektionsfähigkeit unter häuslichen Bedingungen, die in vielen Fällen eine Reduzierung der zu injizierenden Substanz erforderlich machte, führten wir zum einen auf die zu Hause sicherlich adäquatere Stimulation zurück, zum anderen aber auch auf die Untersuchungssituation selbst. Einige Patienten berichteten über eine starke psychische Beeinträchtigung der Erektion duch die dem Penis übergestreiften, sich regelmäßig zusammenziehenden Meßschleifen. Für eine berührungsfreie Dokumentation der Formveränderungen des Gliedes während des Erektionsvorganges, konzipierten wir ein Real-Time-Photogrammetrie-System, das es mit Hilfe stereoskopischer Aufnahmen von Penissilhouetten und digitaler Bildverarbeitung ermöglicht, den Penis dreidimensional zu rekonstruieren und die geometrischen Veränderungen zu analysieren („PRIAPUS“). Die erste praktische Anwendung des Systems an freiwilligen Probanden erfolgte, nachdem Untersuchungen an Penismodellen gezeigt hatten, daß die entsprechenden Parameter für Länge, Durchmesser und Volumen exakt und reproduzierbar bestimmt werden konnten (7). Durch Vergleich mit RigiScan ${ }^{\circledR}$-Rigiditätsaufzeichnungen sollte überprüft werden, ob sich aus den geometrischen Veränderungen berührungsfrei Rückschlüsse auf die Rigidität ziehen lassen

\section{Material und Methode}

Der Aufbau des experimentellen Sichtprüfsystems wurde bereits ausführlich bei den Versuchen mit Penismodellen beschrieben (7). Die Systemsoftware beruht auf einem neuen stereophotogrammetrischen Analyseverfahren, das mit Daten digitalisierter Objektsilhouetten arbeitet. Die Objektsilhouetten werden durch zwei Pulnix TM 840 CCD-Kameras, die das Objekt beobachten, aufgezeichnet. Aus den zwei zweidimensional digitalisierten Bildern wird das Objekt dreidimensional rekonstruiert. Bei der Planung des Versuchsablaufes gingen wir davon aus, daß eine kontinuierliche Aufzeichnung mit einer Bildfrequenz von 15 Sekunden ausreicht, um alle wichtigen Veränderungen des Penis während audiovisueller sexueller Stimulation zu dokumentieren.

Die Positionierung der beiden Kameras erfolgt auf gegenüberliegenden Seiten so, daß die eine Kamera das linke Profil und die andere das rechte Profil des Penis erfaßt. Die optische Achse jeder Kamera muß mit der Horizontalen etwa einen Winkel von 45 Grad bilden. Beide Kameras werden über eine „Video-Junction-Box“ synchronisiert. Das Bild jeder Kamera wird durch einen Matrox-Video-Processor in ein Rasterbild mit $512 \times 480$ Pixeln zu je 8 Bit innerhalb 1/30 Sekunde digitalisiert. Die 8-Bit-Rohbilder werden zunächst über einen Schwellenwert binarisiert und anschließend jene Bildpunkte ermittelt, die die Objektkonturen darstellen. Für die Berechnung der Meßgrößen kommt es vor allem darauf an, die Penisbasis und Penisspitze fehlerfrei zu ermitteln. $\mathrm{Zu}$ diesem Zweck wird angenommen, daß die Penisbasis kreisförmig ist und der Mittelpunkt dieses Kreises den Anfangspunkt der Objektachse darstellt. Das Penisende wird als Halbkugel dargestellt und die Penisspitze definiert als Durchstoßpunkt der Penisachse durch die Oberfläche einer am besten zum Penis passenden Halbkugel. Unter die- sen Voraussetzungen sind Messungen an einem länglichen geraden Objekt, ebenso wie an einem länglichen gekrümmten Objekt, mit hoher Genauigkeit möglich. Aus der dreidimensionalen Zentrallinie wird die Länge des Penis, aus der Silhouette der Durchmesser, und aus Addition der Volumina zylindrischer Scheiben bekannter Länge und bekannten Durchmessers entlang der Objektlänge das Penisvolumen ermittelt.

Da das Meßsystem mit Objektsilhouetten arbeitet, muß sich der Penisrand bei der Aufzeichnung scharf gegen einen Hintergrund abheben. Deshalb wird dem Penis ein schwarzes Lochtuch übergestreift. Nach optimaler Ausleuchtung und Kalibrierung des Systems sind die geometrischen Voraussetzungen zur Aufzeichnung erfüllt.

Bei 30 freiwilligen Probanden, Alter 20-59 Jahre, mit anamnestisch normaler Erektionsfähigkeit wurde während audiovisueller sexueller Stimulation zuerst eine Real-Time-Photogrammetrie des Penis über mindestens 10 Minuten bzw. bis zur maximalen Gliedvergrößerung aufgezeichnet, und anschließend nach einer Pause von 20 Minuten ein RigiScan ${ }^{\circledR}$-Monitoring durchgeführt und die durch die Stimulation maximale Rigidität in Penisschaftmitte bestimmt.

Die für die photogrammetrischen Untersuchungen notwendige Ausleuchtung erfolgte mit drei Beleuchtungskörpern von je 60 Watt, die an einer Aufhängevorrichtung von beiden Seiten den Penis anstrahlten, was von allen Probanden in dieser speziellen Situation als sehr störend empfunden wurde. Zusätzlich führte die Wärmeabstrahlung der Lampen zu einer erheblichen Wärmebelastung.

Nach digitaler Bildverarbeitung wurden die kleinsten und größten Werte für den maximalen $\mathrm{Pe}$ nisdurchmesser, die Penislänge und das Penisvolumen berechnet. Für den Vergleich mit der maximalen Rigidität wurde die jeweilige Größenzunahme benutzt, da die Absolutwerte von der individuell sehr unterschiedlichen Größe des Penis abhängen. Die statistische Bearbeitung der Meßwerte erfolgte mit Hilfe des statistischen Programmes SPSS/PC+. Für die Analyse der Beziehung der Variablen wurde der Pearsonsche Korrelationskoeffizient r berechnet. Für das Signifikanzniveau wurde das $5 \%$ Signifikanzniveau $(\mathrm{p}<0,05)$ zugrunde gelegt.

\section{Ergebnisse}

Vier der 30 Probanden wurden durch die Video-Filme nicht ausreichend stimuliert, so daß bei der RigiScan ${ }^{\circledR}$-Untersuchung lediglich eine Tumeszenzzunahme, aber keine Rigiditätszunahme aufzuzeichnen war. Die Tumeszenz nahm bei allen vier Probanden um weniger als $1 \mathrm{~cm} \mathrm{zu}$. Bei 12 Männern fand sich eine Rigidität $>70 \%$.

Bei allen Probanden, die durch die audiovisuelle Stimulation erregbar waren, trat die Erektion innerhalb der ersten 10 Minuten auf, so daß bei einer Intervallaufzeichnung alle 15 Sekunden jeweils $2 \times 40$ Bilder analysiert wurden. Die einzelnen Meßwerte der Rigidität, 
Tab. 1 RigiScan ${ }^{\circledR}$-Real-Time Ergebnisse (maximale Rigidität) und "PRIAPUS"-Meßwerte bei 30 Probanden. Angegeben sind die Meßwerte der kleinsten Länge, maximalen Durchmesser und Volumen (Min), der größten Länge, maximalen Durchmesser und Volumen (Max) und die berechneten Zunahmen (Diff).

\begin{tabular}{|c|c|c|c|c|c|c|c|c|c|c|}
\hline \multirow[t]{2}{*}{ Proband-Nr. } & \multirow{2}{*}{$\begin{array}{l}\max . \text { Rigidität } \\
(\%)\end{array}$} & \multicolumn{3}{|c|}{ maximaler Durchmesser $(\mathrm{cm})$} & \multicolumn{3}{|c|}{ Länge $(\mathrm{cm})$} & \multicolumn{3}{|c|}{ Volumen $(\mathrm{ccm})$} \\
\hline & & Min & Max & Diff & Min & Max & Diff & Min & Max & Diff \\
\hline 1 & 59 & 2,37 & 5,73 & 3,36 & 3,10 & 11,71 & 8,60 & 10,14 & 91,41 & 81,27 \\
\hline $3^{\star \star}$ & 82 & 2,45 & 3,86 & 1,41 & 8,76 & 16,67 & 7,91 & 27,12 & 110,83 & 83,71 \\
\hline 4 & 10 & 3,13 & 3,43 & 0,30 & 2,71 & 5,55 & 2,84 & 14,30 & 36,14 & 21,85 \\
\hline 5 & 59 & 1,69 & 2,13 & 0,44 & 2,75 & 3,17 & 0,42 & 4,62 & 7,15 & 2,53 \\
\hline $8 * *$ & 86 & 2,91 & 3,58 & 0,67 & 5,97 & 7,56 & 1,59 & 32,49 & 50,41 & 17,92 \\
\hline $9^{*}$ & 0 & 3,21 & 3,35 & 0,14 & 5,94 & 6,94 & 1,00 & 28,94 & 34,73 & 5,80 \\
\hline 10 & 45 & 3,25 & 4,42 & 1,17 & 8,81 & 10,99 & 2,18 & 51,17 & 108,06 & 56,89 \\
\hline 11 & 55 & 3,87 & 4,81 & 0,93 & 8,24 & 10,94 & 2,70 & 74,29 & 128,80 & 54,51 \\
\hline $12^{*}$ & 0 & 2,62 & 3,39 & 0,76 & 3,09 & 4,83 & 1,75 & 16,51 & 27,40 & 10,90 \\
\hline $13^{* *}$ & 89 & 3,88 & 5,75 & 1,87 & 7,23 & 12,90 & 5,68 & 60,88 & 155,24 & 94,36 \\
\hline 19 & 34 & 3,23 & 3,46 & 0,23 & 6,17 & 7,93 & 1,75 & 31,33 & 51,59 & 20,26 \\
\hline $20 * *$ & 82 & 2,89 & 3,88 & 0,99 & 7,74 & 10,48 & 2,74 & 37,00 & 72,68 & 35,69 \\
\hline 21 & 54 & 3,42 & 3,62 & 0,19 & 2,26 & 2,64 & 0,38 & 14,53 & 18,21 & 3,68 \\
\hline $22^{\star}$ & 0 & 3,24 & 4,99 & 1,75 & 1,25 & 2,96 & 1,72 & 5,89 & 17,29 & 11,41 \\
\hline $23^{* *}$ & 93 & 3,17 & 5,63 & 2,45 & 8,09 & 15,15 & 7,06 & 44,86 & 193,55 & 148,69 \\
\hline $24^{* *}$ & 87 & 3,56 & 5,99 & 2,42 & 9,94 & 13,74 & 3,80 & 79,30 & 174,69 & 95,38 \\
\hline 25 & 66 & 3,56 & 6,38 & 2,82 & 9,67 & 13,36 & 3,70 & 60,10 & 151,52 & 91,42 \\
\hline 26 ** & 73 & 3,30 & 4,37 & 1,07 & 8,09 & 10,27 & 2,18 & 50,96 & 73,99 & 23,02 \\
\hline 27 & 62 & 4,34 & 6,69 & 2,35 & 10,38 & 12,82 & 2.44 & 95,23 & 124,76 & 29,53 \\
\hline 28 & 63 & 3,37 & 3,73 & 0,36 & 4,22 & 7,17 & 2,94 & 30,06 & 51,90 & 21,83 \\
\hline $29 * *$ & 82 & 3,21 & 3,96 & 0,75 & 7,21 & 8,72 & 1,51 & 39,03 & 78,05 & 39,02 \\
\hline $30^{*}$ & 0 & 3,14 & 3,36 & 0,22 & 5,76 & 6,75 & 0,99 & 28,24 & 34,76 & 6,52 \\
\hline
\end{tabular}

*Proband ohne Rigidität, ** Probanden mit einer Rigidität $>70$.

Änderung der Penislänge, des maximalen Durchmessers und des Volumens sind in Tab. 1 wiedergegeben. Korreliert wurden die mit der RigiScan ${ }^{\circledR}$-Aufzeichnung ermittelten Werte der maximalen Rigidität mit der photogrammetrisch bestimmten Längen-, maximale Durchmesser- und Volumenzunahme. Die Korrelationsmatrix wurde für alle Probanden (Tab. 2), Probanden mit einer Rigidität $>40 \%$ (Tab. 3), sowie Probanden mit einer Rigidität $>70 \%$ (Tab. 4) berechnet. Zur Überprüfung der Frage, welche Variablen der „PRIAPUS“-Untersuchung die Erektionsfähigkeit determinieren, wurden multiple Regressionsanalysen auf das Kriterium „maximale Rigidität der RigiScan ${ }^{\circledR}$-Aufzeichnung“ durchgeführt. Als Prädiktoren wurden die „PRIAPUS“-Variablen in die Regressionsanalysen eingesetzt. Bei den Regressionsanalysen für alle Probanden $(n=30)$, Probanden mit einer Rigidität $>40 \%(n=22)$ und Probanden mit einer Rigidität $>70 \%$ erfüllte jeweils nur eine Prädiktorvariable die statistischen Kennwerte zur Aufnahme in die Vorhersagegleichung (Tab. 5). In der Gesamtstichprobe und bei Probanden mit einer Rigidität $>40 \%$ wies die Volumenzunahme den größten (linearen) Zusammenhang zur Rigidität auf, bei den Probanden mit einer Rigidität $>70 \%$ dagegen die maximale Durchmesserzunahme, deren Korrelationskoeffizient von 0,695 aber nur geringfügig höher ist als die Korrelation mit der Volumenzunahme $(0,624)$. Die bei 12 Probanden mit einer Rigidität $>70 \%$ ermittelten Werte der Peniskurvatur in
Grad/cm Penislänge sind in Tab. 6 wiedergegeben. Bei dem Probanden Nummer 3 handelt es sich um einen $\mathrm{Pa}$ tienten mit behandlungsbedürftige Penisdeviation $(0,75$ $\mathrm{Grad} / \mathrm{cm}$ ). Die mittlere Deviation der 11 anderen Probanden betrug $0,05 \mathrm{Grad} / \mathrm{cm}$ (SD 0,06).

\section{Diskussion}

Der große Nachteil menschlicher visueller Interpretation besteht in der Subjektivität der Beobachtung. Ein Sichtprüfsystem ist dagegen in der Lage, ein Bild oder eine Bildsequenz objektiv unter einer speziellen Fragestelung zu analysieren. Die digitale Bildverarbeitung ermöglicht es, zu untersuchende Parameter eindeutig und exakt aus Bilddaten zu gewinnen. Nach der Konzeption ei-

Tab. 2 Korrelationsmatrix für alle Probanden $(n=30)$.

\begin{tabular}{lcll}
\hline & $\begin{array}{l}\text { Längen- } \\
\text { zunahme }\end{array}$ & $\begin{array}{l}\text { max. Durchm.- } \\
\text { zunahme }\end{array}$ & $\begin{array}{l}\text { Volumen- } \\
\text { zunahme }\end{array}$ \\
\hline $\begin{array}{l}\text { Rigidität } \\
\text { Längenzunahme } \\
\text { max. Durchm.- }\end{array}$ & 1.00 & .255 & $.539^{*}$ \\
zunahme & .563 & 1.00 & $.805^{* *}$ \\
\end{tabular}


Tab. 3 Korrelationsmatrix für Probanden mit einer Rigidität $>40 \%$ $(n=22)$.

\begin{tabular}{lcll}
\hline & $\begin{array}{l}\text { Längen- } \\
\text { zunahme }\end{array}$ & $\begin{array}{l}\text { max. Durchm.- } \\
\text { zunahme }\end{array}$ & $\begin{array}{l}\text { Volumen- } \\
\text { zunahme }\end{array}$ \\
\hline $\begin{array}{l}\text { Rigidität } \\
\text { Längenzunahme }\end{array}$ & .365 & .041 & $.499^{*}$ \\
$\begin{array}{l}\text { Max. Durchm.- } \\
\text { zunahme }\end{array}$ & .534 & 1.00 & $.534^{\star}$ \\
\hline
\end{tabular}

Tab.4 Korrelationsmatrix für Probanden mit einer Rigidität $>70 \%$ $(n=12)$.

\begin{tabular}{lcll}
\hline & $\begin{array}{l}\text { Längen- } \\
\text { zunahme }\end{array}$ & $\begin{array}{l}\text { max. Durchm.- } \\
\text { zunahme }\end{array}$ & $\begin{array}{l}\text { Volumen- } \\
\text { zunahme }\end{array}$ \\
\hline $\begin{array}{l}\text { Rigidität } \\
\text { Längenzunahme }\end{array}$ & .440 & .695 & $.624^{\star}$ \\
$\begin{array}{l}\text { Max. Durchm.- } \\
\text { zunahme }\end{array}$ & .623 & 1.00 & .623 \\
\hline
\end{tabular}

$* p<0,01 * * p<0,001$

Tab.5 Ergebnis der Regressionsanalyse auf das Kriterium "Rigidität". Gesamtstichprobe $(n=30)$

\begin{tabular}{lll}
\hline Prädikatorvariable & Regressionskoeffizient (Beta)- & R2 \\
\hline Volumenzunahme & .53882 & .29 \\
\hline
\end{tabular}

Probanden mit einer Rigidität $>40 \%(n=22)$

\begin{tabular}{lll}
\hline Prädikatorvariable & Regressionskoeffizient (Beta)- & R2 \\
\hline Volumenzunahme & .49948 & .25
\end{tabular}

Probanden mit einer Rigidität $>70 \%(n=12)$

\begin{tabular}{lll}
\hline Prädikatorvariable & Regressionskoeffizient (Beta)- & $R^{2}$ \\
\hline $\begin{array}{l}\text { maximale } \\
\text { Durchmesserzunahme }\end{array}$ & .69507 & .48 \\
\hline
\end{tabular}

Tab. 6 „PRIAPUS"-Meßergebnisse der Peniskurvatur bei 12 Probanden mit einer Rigidität $>70 \%$.

\begin{tabular}{ll}
\hline Proband-Nr. & Peniskurvatur $(\mathrm{Grad} / \mathrm{cm})$ \\
\hline 2 & 0,102 \\
$3^{*}$ & 0,749 \\
7 & 0,002 \\
8 & 0,015 \\
13 & 0,009 \\
16 & 0,006 \\
17 & 0,180 \\
20 & 0,110 \\
23 & 0,022 \\
24 & 0,014 \\
26 & 0,109 \\
29 & 0,011 \\
\hline
\end{tabular}

* Proband mit behandlungsbedürftiger Penisdeviation nes experimentellen Sichtprüfsystems mit den erforderlichen Handwareprodukten und den notwendigen funktionellen Spezifikationen für Penisaufzeichnungen, wurde in einer Pilotstudie überprüft, ob die Real-Time-Photogrammetrie in der Diagnostik erektiler Dysfunktion sinnvoll anzuwenden ist und als berührungslose Untersuchungsmethode bereits vorhandene Kontaktmethoden ergänzen oder ersetzen kann. Während der klinischen Erprobung mußten zahlreiche Störungen der Bildaufnahme korrigiert werden. Da die Formveränderungen des Penis meßtechnisch durch Änderungen von Grauwerten dokumentiert werden, mußte gewährleistet sein, daß die Grauwertänderungen nicht durch beleuchtungsbedingte Artefakte hervorgerufen werden.

Bei nicht erigiertem kleinen Penis hatte das System Schwierigkeiten, die Penisbasis und Spitze zu ermitteln und die Zentrallinie festzulegen. In diesen Fällen wurde manuell interaktiv die Penisbasis für die Berechnung der Meßparameter markiert. Da der Penis für photogrammetrische Untersuchungen ungünstig, nur ein sehr geringes topographisches Relief und nur wenig Textur für Stereopunktbestimmungen besitzt, war es wichtig die beiden Kameras so zu positionieren, daß ihre beiden optischen Achsen einen Schnittwinkel von etwa 90 Grad bilden. Die Prüfung der Meßgenauigkeit des „PRIAPUS“-Systems an Penismodellen hatte exakte und reproduzierbare Ergebnisse gezeigt (7).

Bei dem Vergleich der Rigidität mit den „PRIAPUS“-Werten wurden standardisiert explizite heterosexuelle Szenen über mindestens 10 Minuten vorgeführt, so daß man von einer für den Großteil der Männer ausreichenden zeitlichen und inhaltlichen Stimulation ausgehen durfte. Trotzdem waren 4 der 30 Probanden, die sich für die vergleichende Untersuchung zur Verfügung gestellt hatten, durch die Filme nicht, und zwei kaum zu stimulieren.

Die RigiScan ${ }^{\circledR}-\mathrm{Me} ß s c h l e i f e$ wurde in Penisschaftmitte angelegt, da Bradley et al. (2) bei 11 potenten Versuchspersonen mit dieser Lokalisation der Schleife eine sehr gute lineare Korrelation der maximalen radialen Rigidität mit der längsgerichteten Kraft ("Buckling Force") nachweisen konnten.

Die vergleichende Untersuchung des Sichtprüfsystems mit der RigiScan ${ }^{\circledR}$-Rigiditätsmessung zeigte bei Erektion eine signifikante Korrelation der Zunahme des maximalen Penisdurchmessers und des Penisvolumens. Dies deutet daraufhin, daß mit der Photogrammetrie eine kontaktlose Beurteilung der Rigidität möglich sein könnte.

Zusätzliche Bestimmungen der Veränderungen des Peniswinkels mit der Horizontalen (Erektionswinkel) durch Implementierung einer dritten Kamera könnten wichtige ergänzende Informationen darstellen, um in Verbindung mit den geometrischen Veränderungen des Penis kontaktlos eine Aussage über die Rigidität treffen zu können.

Erstmals war es mit Hilfe der photogrammetrischen Aufzeichnung, wenn auch nur an einem klei- 
nen Kollektiv möglich, den Normalwert einer noch physiologischen Peniskurvatur mit einer Abweichung des Penis von 0,05 Grad pro cm Länge zu bestimmen. Zur zeit existiert unseres Wissens kein anderes Untersuchungsverfahren, das eine so genaue Dokumentation einer Peniskrümmung ermöglicht. Prä- und postoperative Befunde nach verschiedenen Deviations-Korrekturverfahren könnten mit der Real-Time-Photogrammetrie objektiv verglichen werden.

Der erste Einsatz der Photogrammetrie in der Urologie ist nach den vorliegenden Erfahrungen mit dem experimentellen Sichtprüfsystem „PRIAPUS“ positiv zu bewerten, auch wenn das System zum jetzigen Zeitpunkt die Rigiditätsmessung mit Hilfe dem Penis angelegter Meßschleifen oder Druckaufnehmer noch nicht vollständig ersetzen kann. Entsprechende Anpassungen der Hard- und Software sind erforderlich, um die Untersuchungseinheit zu optimieren.

\section{Literatur}

${ }^{1}$ Bancroft, J., G. Smith, M. Munoz, P. Ronald: Erectile response to visual erotic stimuli before and after intracavernosal papaverine, and its relationship to nocturnal penile tumescence and psychometric assessment, Brit. J. Urol. 68 (1991) 629-638

2 Bradley, W. E., G. W. Timm, J. M. Gallagher, B. K. Johnson: New method for continuous measurement of nocturnal penile tumescence and rigidity, Urology 26 (1985) 4-9

3 Desai, K. M., T. J. Floy, D. H. Follet, D. R. Peake, J. C. Gingell: Development of a penile rigidity indicator and new concepts in the quantification of rigidity, Brit. J. Urol. 61 (1988) 254-260

${ }^{4}$ Frohrib, D. A., I. Goldstein, T. R. Payton: Characaterization of penile erectile states using external computer based monitoring, Trans.A.S.M.E. 109 (1987) 110-114

5 Giesbers, A. A. G. M., J. L. Bruins, A. E. J. L. Kramer, U. Jonas: New methods in the diagnosis of impotence: RigiScan penile tumescence and rigidity monitoring and diagnostic papaverine hydrochlorid injection, World J. Urol. 5 (1987) 173-176

6 Thon, W. F., E. Seidl, H. Freymann, U. Jonas: Erektile Impotenz. Diagnostik und Therapie, Med. Mo. Pharm. 12/10 (1989) $302-305$

7 Thon, W. F., M. Stein, A. E. J. L. Kramer, F. Leberl, U. Jonas: Real-Time-Photogrammetrie in der Urologie. I. Entwicklung eines Sichtprüfsystems für eine berührungsfreie Aufzeichnung von Penisformveränderungen, Akt. Urol. 24 (1993) 28-32.

PD Dr.W.F. Thon

Urologische Klinik

Medizinische Hochschule Hannover

Konstanty-Gutschow Str. 8

3000 Hannover 61 